\title{
Particle swarm optimization applied to solving and appraising the streaming-potential inverse problem
}

\author{
Juan Luis Fernández-Martínez ${ }^{1}$, Esperanza García-Gonzalo ${ }^{2}$, and Véronique Naudet ${ }^{3}$
}

\begin{abstract}
Water flow in the subsoil generates electrical currents measurable at the ground surface with the self-potential (SP) method. These measured potentials, which result from hydroelectric coupling, are called streaming potentials and are well correlated with the geometry of the water table. The particle swarm algorithm can be used to estimate the water-table elevation from SP data measured at the ground surface. The basic idea behind particle swarm optimization (PSO) is that each model searches the model space according to its misfit history and the misfit of the other models (particles) of the swarm. PSO is a simple, robust, and versatile algorithm with a very good convergence rate (typically before 3000 forward runs), and it can explore a large model space without being time consuming. Based on samples gathered in a low-misfit area, we have computed a fast approximation of the posterior distribution of the water table, the electrokinetic coupling constant, and the reference hydraulic head. Although PSO is a stochastic search technique, our convergence results, based on the stability of particle trajectories, specify clear criteria to tune PSO parameters.
\end{abstract}

\section{INTRODUCTION}

Pumping tests are standards methods to estimate the physical properties of aquifers. For this purpose, groundwater flow is monitored with in situ hydraulic-head measurements in a set of observation wells. However, areas of interest typically are sparsely sampled by observation wells because of the high drilling costs. Consequently, nonintrusive geophysical methods that can be implemented over large regions with dense measurements have gained interest in recent years because they can provide complementary information.
One of these methods that is particularly sensitive to groundwater flow is the self-potential (SP) method. Indeed, the flow of groundwater in a porous medium is responsible for a natural electrical field called the streaming potential. This effect results from an electrokinetic phenomenon attributable to the drag of the excess charges present in the diffuse part of the electrical double layer near the water/mineral interface (Onsager, 1931). The drag of excess charges by the groundwater flow is responsible for a net source of electrical current that polarizes the porous medium. The SP method passively records this electrical field at the ground surface with nonpolarizable electrodes. Because the measurement is passive, the recorded signals can be contributed by different natural electrical sources occurring at depth.

The main sources are associated with the hydraulic pressure gradient through the electrokinetic source (the source of interest in our case), the electrochemical sources from ions diffused from concentration gradients between two regions (Maineult et al., 2004), redox processes occurring in orebodies (Bigalke and Grabner, 1997), contaminant plumes (Naudet et al., 2004), corroded metallic casing in boreholes, and measurement noise. To remove the influence of noise and static contributions, the SP survey should be conducted under at least two different flow conditions, e.g., before and during a pumping test. During the last 20 years, there has been renewed interest in applying this method in hydrogeology because it is directly sensitive to groundwater flow, is economically efficient, and is easy to implement in the field.

Many publications show the potential to obtain pertinent hydraulic information on subsurface flows from analyzing SP data (Bogoslovsky and Ogilvy, 1973; Birch, 1998; Sailhac and Marquis, 2001; Revil et al., 2003; Darnet et al., 2004; Bolève, 2007; Minsley et al., 2007; Maineult et al., 2008). These studies interpret directly the streaming-potential anomalies in data space or invert the signals to determine a source model that predicts the data using the problem's underlying physics (forward problem).

Presented at the 78th Annual Meeting, SEG. Manuscript received by the Editor 23 July 2009; revised manuscript received 31 January 2010; published online 30 September 2010.

${ }^{1}$ Formerly University of California Berkeley and Stanford University, California, U.S.A.; presently University of Oviedo, Mathematics Department, Oviedo, Spain.E-mail: jlfm@uniovi.es.

${ }^{2}$ University of Oviedo, Mathematics Department, Oviedo, Spain. E-mail: espe@ uniovi.es.

${ }^{3}$ Université Bordeaux 1, Talence, France, and BRGM (French Geological Survey), Orléans, France. E-mail: v.naudet@brgm.fr.

(C) 2010 Society of Exploration Geophysicists. All rights reserved. 


\section{STREAMING-POTENTIAL GOVERNING EQUATIONS}

We note here the equations governing the electrokinetic problem in a water-saturated porous medium. In steady state and under isothermal conditions, the electric flow $\left(J_{e}\right.$, in $\left.\mathrm{A} / \mathrm{m}^{2}\right)$ is related to the hydraulic pressure gradient $(\nabla P$, in $\mathrm{Pa})$ and the electric potential gradient $(\nabla V$, in $\mathrm{V})$ by the following coupling equation:

$$
J_{e}=\sigma_{r} \nabla V-C_{s} \sigma_{r} \nabla P
$$

where $\sigma_{r}$ is the rock conductivity $(\mathrm{S} / \mathrm{m})$ of the water-saturated porous medium and $C_{s}$ is the electrokinetic coupling coefficient $(\mathrm{V} / \mathrm{Pa})$ of the saturated zone. This coefficient can be measured in the laboratory by applying fluid flow $\Delta P$ and measuring the induced electric potential $\Delta V$ (e.g., Guichet et al., 2006):

$$
C_{s}=\frac{\Delta V}{\Delta P}=\frac{\varepsilon \zeta}{\eta \sigma_{f}} .
$$

Here, $\sigma_{f}$ and $\varepsilon$ are the conductivity and the dielectric constant of the fluid, respectively; $\zeta$ is the zeta potential within the double layer at the interface between the rock and the fluid (Davis et al., 1978; Ishido and Mizutani, 1981; Morgan et al., 1989); and $\eta$ is the dynamic viscosity of the fluid. Most of the time, values of the electrokinetic coefficients in the saturated zone are negative (meaning that positive ions are carried in the flow direction). We consider this coefficient to be homogeneous in the saturated zone and neglected in the unsaturated zone. Indeed, the coupling coefficient is proportional to the water saturation, so it should not be the same in the saturated zone and the unsaturated zone (Revil et al., 2004). Nevertheless, in this paper, we do not account for capillary fringes.

The fundamental equation connecting the electric streaming potential $V$ (in $\mathrm{V}$ ) to the hydraulic pressure $P$ is derived from the conservation of the total electric flux $J_{e}$ defined in equation 1 . In steady state and without direct electric current source, it is written as

$$
\nabla \cdot J_{e}=\nabla \cdot\left(\sigma_{r} \nabla V-C_{s} \sigma_{r} \nabla P\right)=0 .
$$

This is Poisson's equation for $V$, where the divergence of the hydraulic potential gradient is a primary electrokinetic source term. Fitterman (1978) and later Fournier (1989) were the first to consider solutions to Poisson's equation. Revil et al. (2003) use Fournier's first integral formulas to model the streaming potential caused by aquifers in tabular media. They show that in steady-state conditions, the piezometric surface (i.e., the water table) can be assimilated mathematically to a dipole layer of charge with strength proportional to the piezometric head, obtaining the expression for the electric potential measured at an observation station $Q$ located at the earth's surface:

$$
V(Q)=\frac{C^{\prime}}{2 \pi} \int_{\partial \Omega}\left(h-h_{0}\right) \frac{\mathbf{r} \cdot \mathbf{n}}{\|\mathbf{r}\|^{3}} d \Gamma+\frac{1}{4 \pi} \int_{\Omega} \frac{\mathbf{E}}{\|\mathbf{r}\|} \cdot \frac{\nabla \rho}{\rho} d V,
$$

where $\mathbf{n}$ is the outward normal to the water table; $h$ and $h_{0}$ are, respectively, the hydraulic heads at any point of the hydraulic head and in a reference level; $\mathbf{r}$ is the vector from any source point in the hydraulic head to observation station $Q ; \mathbf{E}=-\nabla \phi$ is the electrical field produced in the ground through the electrokinetic coupling; $\rho$ is the electrical resistivity of the rock (in ohm-m); and $C^{\prime}$ (in $\mathrm{mV} / \mathrm{m}$ ) is the electrokinetic coupling coefficient relating the hydraulic piezometric head $\Delta h(\mathrm{~m} / \mathrm{s})$ to the electrical potential difference $\Delta V$ (in $\mathrm{mV}$ ). The relationship between $C_{s}$ (equation 2) and $C^{\prime}$ is $C^{\prime}=\rho_{f} \mathbf{g} C_{s}$, with $\rho_{f}$ the mass density (in $\mathrm{kg} / \mathrm{m}^{3}$ ) and $\mathbf{g}$ the gravity acceleration vector (in $\mathrm{m} / \mathrm{s}^{2}$ ).

In this model, the distribution of the electric potential is the sum of two terms. The first term is related to the current density (primary source of polarization), and the second one is related to electrical-resistivity contrasts in the ground (secondary source of polarization). To separate these contributions and focus on the electrokinetic source, different strategies can be performed. For example, electrical-resistivity tomography can be performed onsite to provide the electrical structure of the ground and incorporate the information into the inversion. To remove the other static contributions (electrode noise, telluric noise, regional trend, etc.), the SP survey must be conducted under at least two different flow conditions, such as before and during the pumping test.

The primary source term is such that each element of the water table acts as a dipole of strength $C^{\prime}\left(h-h_{0}\right)$. All of these dipoles contribute to the streaming-potential signals recorded at $Q$, with strength also depending on the distance between each dipole and $Q$. In our case, the aim is to recover the depth of the water table and $C^{\prime}$ from the SP measurements performed at the ground surface. Thus, the rest of the sources on the SP signal will be considered corrections of the primary effect. In other words, the algorithm presented here applies to the part of the SP signal that results only from the electrokinetic primary source, i.e., the streaming potential. We are particularly focused on inverting SP anomalies associated with ground flow from pumping tests.

Several algorithms have been applied to invert SP data. Patella (1997) and Revil et al. (2003) use correlation integrals to locate SP sources underground. Other theories, also based upon classical convolution integrals of potential field theory, invert SP data in the wavelet domain to identify location, intensity, and type of singularities of causative underground hydraulic flows (Gibert and Pessel, 2001; Sailhac and Marquis, 2001). Rizzo et al. (2004) invert the SP signal associated with pumping-test experiments using the theory of Green's functions, naming the new methodology electrography. Also, Revil et al. (2003) use a simplex algorithm to invert Fournier's equation for an unconfined aquifer in a medium of constant electric conductivity. Its main drawback is its very slow convergence. Minsley (2007) and Sheffer and Oldenburg (2007) consider a heterogeneous electric-conductivity distribution, observed using electrical resistivity tomography (ERT) to invert the electric Poisson equation and obtain a tomogram of SP source intensity. Typically, the preferred method to perform these kinds of inversions is standard least squares with Tikhonov regularization.

The hydrogeophysical community is increasingly interested in quantifying the uncertainty of estimation. Bayesian approaches and global-optimization methods are used to accomplish this task. Linde et al. (2007) apply a Bayesian method using geostatistical techniques to integrate SP and piezometric data to estimate the water table throughout a catchment. Jardani et al. (2009) propose an algorithm to solve the SP inverse problem in a Bayesian framework. According to our knowledge, the use of global algorithms has been restricted to genetic algorithms (Darnet et al., 2003). The reason might be that research has been focused on the underlying physical theory and solving the forward problem rather than on the inverse problem 
algorithm itself. Nevertheless, both are critical to achieve good solutions.

We present the application of the particle swarm optimization (PSO) algorithm to estimate the depth of the water table from SP data, expanding and clarifying the methodology presented in Naudet et al. (2008). We first detail how PSO works and present results of our recent research about the criteria used to tune PSO parameters to achieve algorithm convergence. Afterward, the algorithm is tested on synthetic and field SP data collected near a pumping well (Bogoslovsky and Ogilvy, 1973). Synthetic modeling shows us that the topography of the cost function corresponds to a flat valley shape. Additionally, we analyze the equivalences between the amplitude and dispersion of a Gaussian hydraulic head and the electrokinetic coupling coefficient. We also show that PSO is very robust in the presence of noisy data. The reason is that the inverse problem is not solved as an optimization problem but as a sampling problem. When applied to field data, correlation length is a critical parameter to recover different families of geophysical models having different regularity. Nevertheless, not all of them are compatible with borehole data.

\section{GLOBAL OPTIMIZATION METHODS AND SAMPLING}

Most inverse problems can be written in discrete form as

$$
\mathbf{d}=\mathbf{F}(\mathbf{m}), \quad \mathbf{d} \in R^{s}, \quad \mathbf{m} \in R^{n},
$$

where $\mathbf{d}$ is the observed data, $\mathbf{m}$ is the vector containing the model parameters, and $\mathbf{F}$ is the physical model. Given a particular observed data set $\mathbf{d}_{0}$, the goal of inversion is to find a set of reasonable parameters $\mathbf{m}_{0}$ such that $\mathbf{F}\left(\mathbf{m}_{0}\right)=\mathbf{d}_{0}$. Usually this problem has no solution or many solutions; thus, the solution of the inverse problem must be constructed (Parker, 1994; Tarantola, 2004; Aster et al., 2005). The inverse problem is then solved as an optimization problem - finding $\mathbf{m}_{0}$ that minimizes the data-prediction error, expressed in a certain norm $\|\mathbf{d}-\mathbf{F}(\mathbf{m})\|_{p}$.

This optimization problem turns out to be ill posed for three reasons. First, the forward model $\mathbf{F}$ is a simplification of reality. Second, data are noisy and only partially sample the domain of interest. Third, most applications of inverse modeling do not include a priori knowledge of physical constraints or the amount and quality of available data in the inversion. These three points cause an inverse problem to be very different from any other kind of optimization problem because physics and data are involved in the cost function $\|\mathbf{d}-\mathbf{F}(\mathbf{m})\|_{p}$.

Although a variety of optimization algorithms exist in the scientific literature, how to select the appropriate algorithm to solve an inverse problem remains an active subject of research. These methods involve well-known techniques such as linear algebra, local and global optimization, Bayesian approaches, neural networks, Kalman filters, kernel methods, and support vector machines. In addition, finding the global minima of the error function is often complicated by the presence of many local minima and/or the error-space topography surrounding the global minima is very flat and oblong. Primarily, the nature of error-space topography is dependent upon the forward model, or physics. Additionally, data noise can increase the presence of local minima and/or the size of the valley topography (see, e.g., Fernández Alvarez et al., 2008; Fernández Martínez and Pedruelo González, 2008).

Local-optimization methods are unable to discriminate among the multiple choices consistent with the end criteria and may land quite unpredictably at any point in that area. These pathologies are treated through regularization techniques and the use of good prior information and/or initial guesses.

Once the inverse problem is solved, an important issue is the model appraisal to quantify the uncertainty of the estimate. When using local-optimization algorithms, the appraisal is usually done through linearizing the inverse problem functional F. Bayesian approaches and Monte Carlo methods (Mosegaard and Tarantola, 1995; Scales and Tenorio, 2001) can solve the inverse problem in a different way, implicitly accomplishing the model-appraisal task. In this case, the inverse problem is formulated such that the posterior probability distribution is sampled many times in a random walk, with a bias toward increased sampling of areas of higher probability to approximate the whole posterior probability accurately (so-called importance sampling). The drawback of such approaches is that they are computationally expensive and might not be feasible in case of slow forward problems.

Posterior sampling techniques are closely related to global-optimization algorithms, which can be used to provide a proxy for the true posterior distribution. In many practical situations, prior information is unavailable, so global-optimization methods are a good alternative for avoiding the strong dependence of the solution on noisy data and on the initial guess. Most of these techniques are stochastic and heuristic, and they try to sample the low-misfit region of model parameters, i.e., region $\mathbf{E}$ of model space $\mathbf{M}$ containing the models that fit the observed data within a given tolerance. As mentioned, this sampling procedure acquires the full sense in a Bayesian framework. Nevertheless, independent of the name that practitioners use to classify the inversion procedure, the main mechanism involved is sampling.

One of the peculiarities of global algorithms is that they do not try to solve the inverse problem as an optimization problem but as a sampling problem; thus, they do not need a prior model to stabilize the inversion. In fact, the only prior information these algorithms need is to specify the search space, which can be very large if no prior information is available. In a few cases, the space of solutions is so large that some form of regularization that constrains the solution sampling to a range of geologically acceptable limits is necessary.

Global-optimization algorithms include well-known techniques such as genetic algorithms (Holland, 1992), simulated annealing (Kirkpatrick et al., 1983), PSO (Kennedy and Eberhart, 1995), differential evolution (Storn and Price, 1997), and the neighborhood algorithm (Sambridge, 1999a). Some examples of the applications of these methods for finding and evaluating solutions to geophysical inverse problems are provided by Stoffa and Sen (1991) and Fernández Álvarez et al. (2008) for genetic algorithms; Sen and Stoffa (1995) and Ma (2002) for simulated annealing; Shaw and Srivastava (2007), Fernández-Martínez et al. (2008a), Naudet et al. (2008), and Sanyi et al. (2009) for PSO; and Fernández-Martínez et al. (2009) for differential evolution.

Under certain conditions, global algorithms can provide accurate measures for model uncertainty. Mosegaard and Tarantola (1995) used simulated tempering to perform importance sampling of model parameters with the Metropolis-Hastings algorithm. More recently, Fernández Álvarez et al. (2008) show numerically the ability of binary genetic algorithms to perform an acceptable sampling of the 
posterior distribution when used for exploration. The neighborhood algorithm can also be used to accomplish this task (Sambridge, 1999b). Although PSO has not been designed to perform importance sampling, it provides a proxy for the posterior distribution for the model parameters in its exploitative form (Fernández-Martínez et al., 2009). Nevertheless, PSO has the main advantage of being a very fast sampler and shows good balance between exploration and convergence.

\section{PARTICLE SWARM OPTIMIZATION}

PSO is a stochastic evolutionary computation technique (Kennedy and Eberhart, 1995) that has been used successfully in many different engineering fields. It is inspired by the social behavior of individuals (called particles) in nature, such as birds flocking and fish schooling. Each particle samples the search space according to its own $\mathbf{l}_{i}(k)$ and its companions' $\mathbf{g}(k)$ searching experience.

The way the PSO algorithm is applied to solve an inverse problem is very intuitive. A prismatic space of admissible geophysical models $\mathbf{M}$ is defined:

$$
\ell_{j} \leq x_{j i} \leq u_{j}, \quad 1 \leq j \leq n, \quad 1 \leq i \leq N_{\text {size }},
$$

where $\ell_{j}$ and $u_{j}$ are the lower and upper limits for the $j$ th coordinate of each geophysical model (called $i$ ), $n$ is the number of parameters in the inverse problem, and $N_{\text {size }}$ is the swarm size. In PSO terminology, each geophysical model is called a particle, represented by a vector whose length is the number of degrees of freedom of the inverse problem. Each particle has its own position on the search space and velocity. The velocities are the parameter perturbations needed for these positions to find the solutions of the inverse problem.

The algorithm updates at each iteration the positions $\mathbf{x}_{i}(k)$ and velocities $\mathbf{v}_{i}(k)$ of each model in the swarm. The velocity of each particle $i$ at each iteration $k$ is a function of three major components. The first is the inertia term, which consists of the old velocity vector of the particle weighted by a real constant $\omega$ called inertia. The second is the social learning term, which is the difference between the global best position found thus far in the entire swarm $\mathbf{g}(k)$ and the particle's current position. The third component is the cognitive learning term, which is the difference between the particle's best position found so far, $\mathbf{l}_{i}(k)$, and the particle's current position.

These three components are represented numerically as

$$
\begin{aligned}
& \mathbf{v}_{i}(k+1)=\omega \mathbf{v}_{i}(k)+\phi_{1}\left(\mathbf{g}(k)-\mathbf{x}_{i}(k)\right)+\phi_{2}\left(\mathbf{l}_{i}(k)-\mathbf{x}_{i}(k)\right), \\
& \mathbf{x}_{i}(k+1)=\mathbf{x}_{i}(k)+\mathbf{v}_{i}(k+1),
\end{aligned}
$$

where PSO parameters $\omega, a_{g}$, and $a_{\ell}$ are inertia and the local and global acceleration constants, respectively; $\phi_{1}=r_{1} a_{g}$ and $\phi_{2}=r_{2} a_{\ell}$ are the stochastic global and local accelerations; and $r_{1}$ and $r_{2}$ are vectors of random numbers uniformly distributed in $(0,1)$ to weight the global and local acceleration constants $a_{g}$ and $a_{\ell}$ of any coordinate particle in the swarm. The total mean acceleration $\bar{\phi}=\left(a_{g}+a_{\ell}\right) / 2$, along with the inertia constant $\omega$, plays an important role in the PSO parameter selection to achieve convergence.

To summarize, the stepwise process for the PSO algorithm is as follows:

1) Give as prior information a prismatic search space.

2) Uniformly distribute an initial swarm of $N_{\text {size }}$ particles in the search space and set their initial velocities to zero (usually).

3) Evaluate the misfit of the initial population, solving $N_{\text {size }}$ for- ward problems and determining the global best and previous best of each particle.

4) Draw the random numbers $r_{1}$ and $r_{2}$, and update the velocities and positions of each particle of the swarm using formula 7 .

5) Iterate to point 3 until the maximum number of iterations is finished or some stop criteria are fulfilled.

\section{PSO physical model and generalized PSO}

The PSO algorithm can be interpreted physically as a stochastic damped mass-spring system (Fernández-Martínez et al., 2008b). PSO corresponds to a particular discretization of the differential equation:

$$
x^{\prime \prime}(t)+(1-\omega) x^{\prime}(t)+\left(\phi_{1}+\phi_{2}\right) x(t)=\phi_{1} g(t)+\phi_{2} \mathbf{l}(t) .
$$

Equation 8 describes the continuous movement of each particle in the swarm. PSO corresponds to a centered discretization in acceleration,

$$
x^{\prime \prime}(t)=\frac{x(t+\Delta t)-2 x(t)+x(t-\Delta t)}{\Delta t^{2}},
$$

and a regressive (backward) schema in velocity,

$$
x^{\prime}(t)=\frac{x(t)-x(t-\Delta t)}{\Delta t} .
$$

This result allows us to propose the generalized PSO (GPSO) algorithm (Fernández Martínez and García Gonzalo, 2008), which is a PSO generalization for any time step $\Delta t$ :

$$
\begin{aligned}
v(t+\Delta t)= & (1-(1-\omega) \Delta t) v(t)+\phi_{1} \Delta t(g(t)-x(t)) \\
& +\phi_{2} \Delta t(\mathbf{l}(t)-x(t)), \\
x(t+\Delta t)= & x(t)+\Delta t v(t+\Delta t) .
\end{aligned}
$$

Using $\Delta t=1$, equation 11 reduces to the PSO algorithm (equation 7). As a result of the random effect introduced by $r_{1}$ and $r_{2}$, the particle trajectories must be considered as stochastic processes whose first- (mean) and second-order moments (variance and temporal covariance) are important in understanding algorithm convergence.

The first-order stability region is the part of the $\left(\omega, a_{g}, a_{\ell}\right)$ space where the mean particle trajectories are stable. For the GPSO case, the first-order stability region is

$$
\begin{aligned}
S_{1} & =\left\{\left(\omega, a_{\ell}, a_{g}\right): 1-\frac{2}{\Delta t}<\omega<1,0<\bar{\phi}<\phi_{h}\right. \\
& \left.=\frac{2(\omega-1) \Delta t+4}{\Delta t^{2}}\right\} .
\end{aligned}
$$

For any $\Delta t$, this region generalizes the first-order stability region found for the PSO case (Clerc and Kennedy, 2002; Trelea, 2003).

Choosing the PSO parameters $\left(\omega, a_{g}, a_{\ell}\right)$ such that point $(\omega, \bar{\phi})$ lies inside the first-order stability region causes the particle trajectories to converge toward the oscillation center $\mathbf{o}_{i}(k)$ : 


$$
\mathbf{o}_{i}(k)=\frac{a_{g} \mathbf{g}(k)+a_{\ell} \mathbf{l}_{i}(k)}{a_{g}+a_{\ell}} .
$$

The second-order stability region is the part of $S_{1}$ where the second-order moments $E\left(x_{i}^{2}(k)\right), E\left(x_{i}^{2}(k+1)\right)$, and $E\left(x_{i}(k) x_{i}(k+1)\right)$ remain stable. Fernández Martínez and García Gonzalo (2008) show that the second-order stability zone in the GPSO case can be written as

$$
\begin{aligned}
& S_{2}=\left\{\left(\omega, a_{l}, a_{g}\right):-1<\omega<1,0<\bar{\phi}<\phi_{h}\right\}, \\
& \phi_{h}=\frac{12}{\Delta t} \frac{(1-\omega)(2+(\omega-1) \Delta t)}{4-4(\omega-1) \Delta t+\left(\alpha^{2}-2 \alpha\right)(2+(\omega-1) \Delta t)},
\end{aligned}
$$

where $\alpha=a_{g} / \bar{\phi}$ is the ratio between the global and total mean acceleration, and it varies in the $[0,2]$ interval. Low values of $\alpha$ with the same value of $\bar{\phi}$ imply that the local acceleration is bigger than the global one and, thus, the algorithm is more explorative. The secondorder stability region is embedded within the region of first-order stability. The hyperbola of second-order stability $\bar{\phi}=\phi_{h}$ marks the limit where the variance of the trajectories conceived as stochastic processes becomes unbounded. Thus, the region close to this line is an area of high exploration capabilities (Fernández-Martínez et al., 2008b).

Knowledge of both stability regions is very important to establish sufficient conditions for the PSO convergence. If the inverse problem is well posed, i.e., the global minimum is unique, a sufficient condition for convergence is that the oscillation center approach with iterations the global minimum and attract the particles toward its position. This is sure to happen when the PSO parameters are chosen within the region of second-order stability. Nevertheless, some $\left(\omega, a_{\ell}\right.$, $a_{g}$ ) points are better than others to perform this task. In the case of multimodal functions, entrapment in local minima is also possible. For this reason, it is very important to have algorithms with a good balance between exploitation and exploration.

\section{How to tune PSO parameters}

As a result of numerical experiments with benchmark functions and theoretical studies to understand the PSO convergence, three popular parameter sets have been proposed: Carlisle and Dozier's point (2001), $\left(\omega, a_{g}, a_{\ell}\right)=(0.729$, $0.948,2.041)$; Clerc and Kennedy's point (2002), $\left(\omega, a_{g}, a_{\ell}\right)=(0.729,1.494,1.494) ;$ and Trelea's point $(2003),\left(\omega, a_{g}, a_{\ell}\right)=(0.6,1.7,1.7)$.

Although by using these parameters the scientific community could solve a variety of engineering problems (Poli, 2008), tuning the PSO parameters has been a weak point in the application. Fernández Martínez and García Gonzalo (2008, 2009) show that no optimal PSO parameters (which are highly dependent on the kind of cost function) exist. Instead, good PSO parameters are found for a broad range of cost functions in regions where PSO has a higher probability to reach good solutions. Figure 1 shows contour plots of the percentage of times the GPSO algorithm provides a solution located in the neighborhood of the global minimum after 100 iterations and the median errors (in this case, the position of the global minimum is known) expressed on a logarithmic scale for the Rosenbrock (flat valley topography) and Griewank (many local minima) functions. This numerical analysis is performed for a $(\omega, \bar{\phi})$ grid that includes the first-order stability region. The most successful $(\omega, \bar{\phi})$ points found in the literature are also shown. The points are close to the intersection of the upper limit of second order and the median of the stability triangle, $\bar{\phi}=(2-(1-\omega) \Delta t) / \Delta t^{2}$. It is possible to show that along this line, the temporal correlation between $x(t)$ and $x(t+\Delta t)$ goes to zero very fast (typically before 10 iterations). In conclusion, good $(\omega, \bar{\phi})$ parameter sets are located on a region of maximum exploration inside the second-order stability region.

\section{Exploration versus exploitation}

PSO can be viewed not as a unique algorithm but as a set of algorithms that can be used for exploitation (looking for a unique global minimum) and/or exploration (sampling the low-misfit region in the model space). The design of the PSO version (explorative or exploitative) depends on two major factors. The first factor is the $\left(\omega, a_{g}, a_{\ell}\right)$ point chosen. As explained, the greatest explorative behavior is achieved when $\left(\omega, a_{g}, a_{\ell}\right)$ is close to or even below the second-order stability upper limit. Also, for the same total mean acceleration $\bar{\phi}$ $=\left(a_{g}+a_{\ell}\right) / 2$, choosing $a_{g}<a_{\ell}$ makes the algorithm more explorative.

The second factor is the $\Delta t$ parameter, which is a numerical constriction factor to achieve stability. It is possible to show analytically that the first- and second-order stability regions increase their size
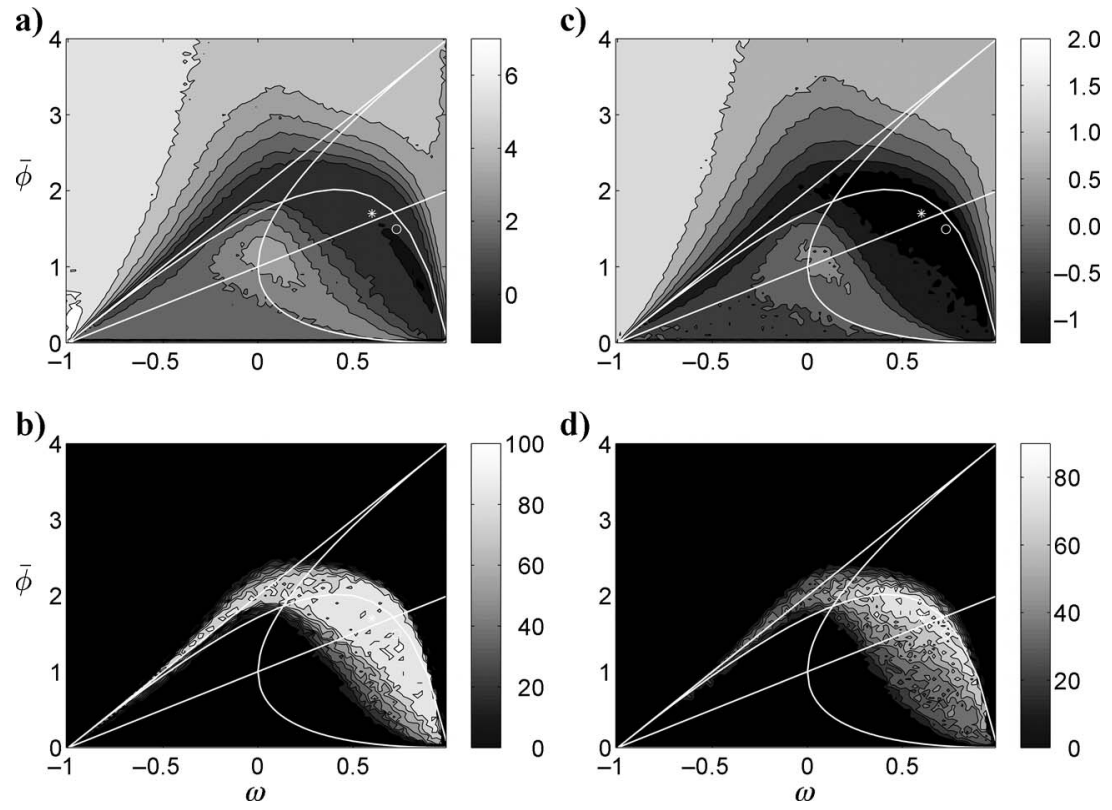

Figure 1. Median error (logarithmic scale, top row) and percentage of successes (bottom row) for the (a, b) Rosenbrock (flat valley topography) and (c, d) Griewank (many local minima) functions. Observe the triangle of first-order stability, the hyperbola of secondorder stability, and the median line of temporal uncorrelation between trajectories. Asterisks show the Trelea point; circles show the Clerc and Kennedy point. 
and tend toward $\{\omega<1, \bar{\phi}>0\}$ when $\Delta t$ goes to zero. In this case, the exploration is increased around the global best solution. Conversely, when $\Delta t$ is greater than one, the first- and second-order stability regions shrink in size and the exploration is increased in the whole search space. This feature might help avoid entrapment in local minima and has inspired us to create the lime and sand algorithm that alternates values of $\Delta t$ greater and less than one, depending on the iterations (Fernández Martínez and García Gonzalo, 2008).

Based on these results, García Gonzalo and Fernández Martínez (2009) have designed a PSO algorithm where each particle in the swarm has different inertia (damping) and acceleration (rigidity) constants. This algorithm, the PSO cloud, exhibits good balance between exploration and exploitation. The results obtained for hard benchmark functions in several dimensions using the PSO cloud are better than the reference misfits for PSO in the literature. This design avoids two main drawbacks of the PSO algorithm: tuning the PSO parameters and clamping the velocities. The criteria for choosing the cloud points are not very rigid because points close to the second-order convergence border achieve good results (low-misfit areas shown in Figure 1).

\section{How to input prior information}

It is well established in optimization theory that different norms and penalizations defined in the cost function reveal different kinds of solutions with different regularity. For instance, the $\ell_{1}$-norm allows searching for sparse and blocky solutions. Conversely, soft solutions can be found using the $\ell_{2}$-norm, introducing as penalization some regularity requirements in the model.

In the PSO case, soft solutions are found in a very simple way by smoothing the global best, providing to the algorithm an attractor with a prescribed smoothness. Reference models can be input very easily into the PSO algorithm through at least three different ways. One way is as a particle that is introduced occasionally into the swarm. If the reference model is compatible with the data, the reference model might influence the oscillation center. A second way is through the misfit functional, including a regularization term:

$$
c(\mathbf{m})=w_{1}\|\mathbf{d}-\mathbf{F}(\mathbf{m})\|_{p}+w_{2}\left\|\mathbf{m}-\mathbf{m}_{\mathrm{ref}}\right\|_{p^{\prime}},
$$

where $w_{1}$ and $w_{2}$ are real weights. Finally, the model can be introduced as an attractor $\phi_{3} \mathbf{m}_{\text {ref }}$ into the force term. There are two variants in this case: it can be considered as a third attractor term,

$$
\begin{aligned}
v(t+\Delta t)= & (1-(1-\omega) \Delta t) v(t)+\phi_{1} \Delta t(g(t)-x(t)) \\
& +\phi_{2} \Delta t(\mathbf{l}(t)-x(t))+\phi_{3} \Delta t\left(m_{\mathrm{ref}}-x(t)\right), \\
x(t+\Delta t)= & x(t)+\Delta t v(t+\Delta t),
\end{aligned}
$$

or $\phi_{3} \Delta t\left(m_{\text {ref }}-x(t)\right)$ might replace the global best attractor $\phi_{1} \Delta t(g(t)-x(t))$ at the end of the algorithm execution. In all three cases, the reference model $\mathbf{m}_{\text {ref }}$ has to be a vector with the same dimensions as the swarm particles.

In the study case shown in the next section, no prior model was used, and the Euclidean was the norm for misfit calculations.

\section{Peculiarities of PSO design in the streaming-potential case}

Although PSO is a general-purpose algorithm, its successful application to real problems consists partly in understanding the phys- ics involved in the forward problem and adapting the PSO algorithm to the inverse-problem's peculiarities.

In the SP inverse problem, the parameters optimized by the PSO algorithm are the piezometric heads $h_{i}$, the electrokinetic coupling coefficient $C^{\prime}$, and the reference hydraulic head $h_{0}$, which usually is unknown. We assume that the resistivity structure is homogeneous and that the electrokinetic coupling coefficient is homogeneous in the saturated zone. Thus, the number of parameters is $n_{\text {node }}+2$, where $n_{\text {node }}$ is the number of discretization nodes used to interpolate the hydraulic head. This allows the problem not to be underdetermined.

PSO constrains the search domain to optimize the parameters. For the upper and lower limits of $C^{\prime}$, it is recommended to use a very broad interval at the exploratory stages, such as $[-20,20] \mathrm{mV} / \mathrm{m}$. Based on the SP anomaly, it is also convenient to establish the sign of the $C^{\prime}$ constant to improve search efficiency.

To establish the lower and upper limits for the piezometric levels, we can establish a prismatic search space or we can use the semiempirical relationship proposed by Revil et al. (2003):

$$
V(P)=\left(h-h_{0}\right) C^{\prime} .
$$

The upper and lower limits $h_{\max }$ and $h_{\min }$, respectively, are therefore defined as

$$
\begin{aligned}
& h_{\text {min }}(P)=h_{0}+\frac{V(P)}{C_{\text {max }}^{\prime}}, \\
& h_{\text {max }}(P)=h_{0}+\frac{V(P)}{C_{\text {min }}^{\prime}} .
\end{aligned}
$$

Note that formula 18 cannot be used when the variability associated with $C^{\prime}$ is very tight. This might happen when we want the value of the electrokinetic parameter to be prescribed.

The PSO version used for SP optimization has some important peculiarities. First, when the number of parameters increases (from a finer discretization of the water table), better results are achieved by giving greater weight to the global best than to the local best (the global leader must be the main attractor) to succeed in locating the global minimum area. Also, the best parameter sets move toward a region of higher inertias and lower total mean accelerations where the temporal covariance between trajectories is positive (see Figure 1). Another peculiarity is that the SP inverse problem is ill posed, i.e., very different geophysical models exhibiting very different spatial correlation lengths can account for the observed data within the same error tolerance. The hydraulic-head parameters are not spatially independent; to improve the exploration/convergence task, the global best is smoothed to allow for gradual changes in the water table. As we show in the field data case, an estimate of the global best smoothness length can be deduced from the autocorrelation function of the SP data.

\section{APPLICATION OF PSO TO SYNTHETIC AND REAL DATA}

The aim of this section is to test PSO performance on a synthetic data case for different levels of Gaussian noise and to analyze the topography of the cost function to identify which kinds of pathologies we might expect in the optimization process.

We first simulate the SP data associated with a pumping test in steady-state conditions. Pumping in a well causes a depression cone 
of the water table. In response to steady-state pumping, a positive SP anomaly is usually observed as a result of electrokinetic coupling. For our synthetic cases, the water table is modeled by a Gaussian function:

$$
h\left(x ; A, \sigma, x_{w}\right)=A e^{-\left(x-x_{w}\right)^{2} / 2 \sigma^{2}} .
$$

In this case, $A$ is the drawdown amplitude, $\sigma$ is the dispersion around the central value $A$, and $x_{w}$ is where the pumping test is performed, fixed to $200 \mathrm{~m}$. Here, the coupling coefficient has been set up to $C^{\prime}$ $=-5 \mathrm{mV} / \mathrm{m}$, and the reference hydraulic head $h_{0}$ used to generate the synthetic data is $45 \mathrm{~m}$.

We use synthetic models to analyze the equivalences of the model parameters $\left(A, \sigma, C^{\prime}\right)$ by varying two of the three parameters at the same time and studying the algorithm performance as a function of the noise level. For this case, the true model was $\left(A, \sigma, C^{\prime}\right)=$ $(20,40,-5)$. Figure 2 a shows the topography of the error function in the vicinity of $(A, \sigma)=(20,40)$ when $C^{\prime}$ is set to the true value. To produce this contour plot, we calculate the forward problem for a lattice of different points $\left(A_{k}, \sigma_{k}\right)$ in the vicinity of the corresponding true model. The relative error between the prediction of true and perturbed models is calculated with the $\ell_{2}$-norm. The error function has a valley shape around the global minimum area. Figure $2 \mathrm{a}$ also includes the $3 \%$ and $10 \%$ error isolines. The correlation between these parameters is negative.
Figure $2 \mathrm{~b}$ shows the topography of the error function in the vicinity of $\left(A, C^{\prime}\right)=(20,-5)$, and Figure $2 \mathrm{c}$ shows the topography of the error function near $\left(\sigma, C^{\prime}\right)=(40,-5)$. The correlation between these parameters is positive. Considering the size of the $10 \%$ error region shown in the three cases, the most important trade-off appears to be between the drawdown amplitude and the coupling constant. These features might explain the existing trade-offs in real cases. Also, with noisy real data, the valley increases in size and the presence of localized sinkholes might provoke entrapment in local minima. Figure 2d-f explores the equivalences between $h_{0}, A, \sigma$, and $C^{\prime}$. The most important trade-off is with the amplitude of the water table. The two relations with $\sigma$ and $C^{\prime}$ seem to be resolved quite well.

Figure 3 shows the results predicted for a synthetic case corresponding to $\left(A, \sigma, C^{\prime}\right)=(20,40,-5)$ when the data are free of noise. The number of discretization points (pixels) for the hydraulic head has been set equal to the number of data points minus one. The swarm size and the number of iterations are set to 100 . The search space is $C^{\prime} \in[-20,1], h \in[0.1,30]$. Figure 3 also shows the true model used to generate the synthetic SP data and the inverted model having the lower misfit. The SP observed anomaly is almost perfectly matched. The PSO methodology allows sampling-promising regions of the model space, thus making a posteriori statistics. Figure $3 \mathrm{a}$ also shows the median of the models found in the $10 \%$ error region and the lower and upper bounds, using the interquartile range calculated from the posterior statistics. In this case, the true model is closer to the lower bound. In other simulations that we have performed, the true model is within the lower and upper water-table a)

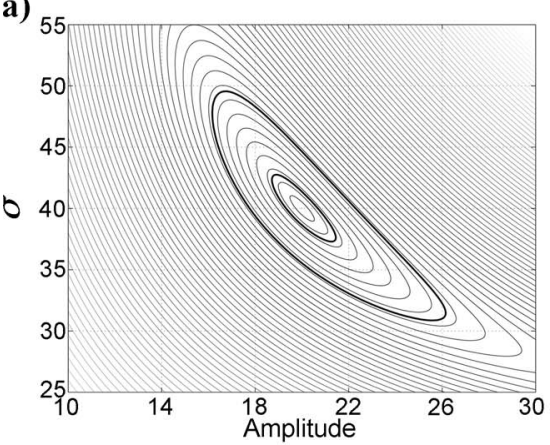

d) ${ }_{47}$

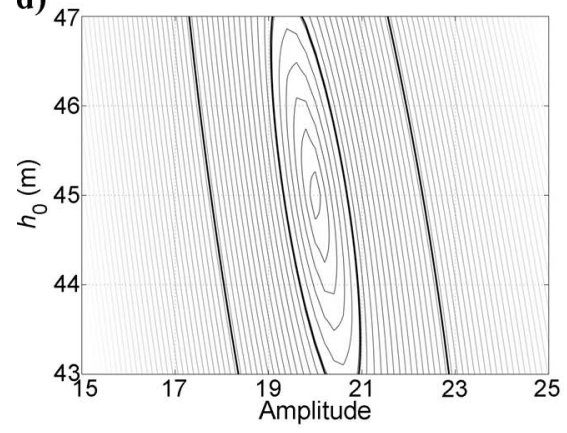

b)

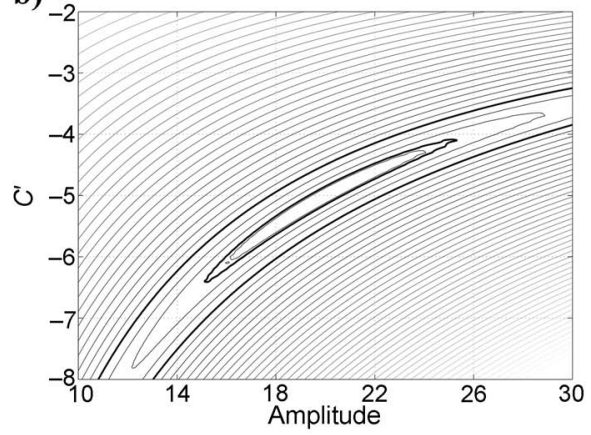

e)

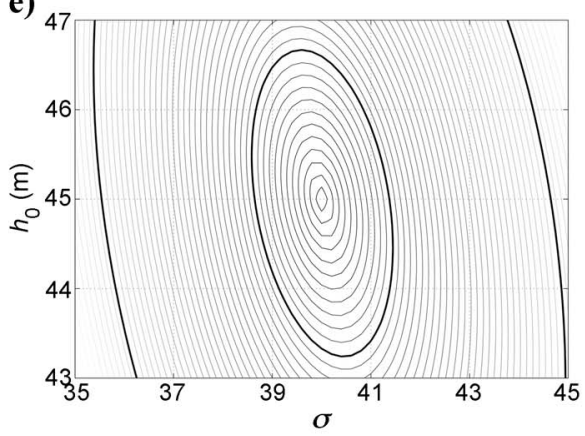

c)

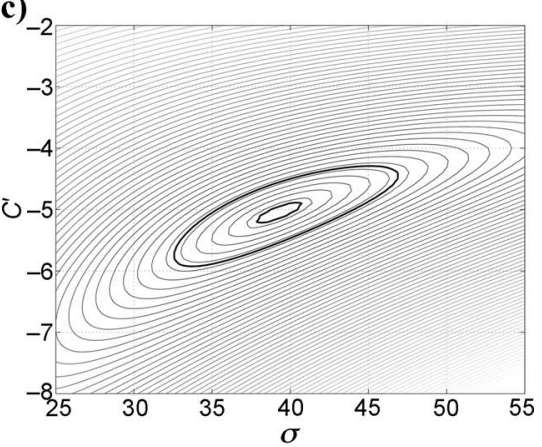

f)

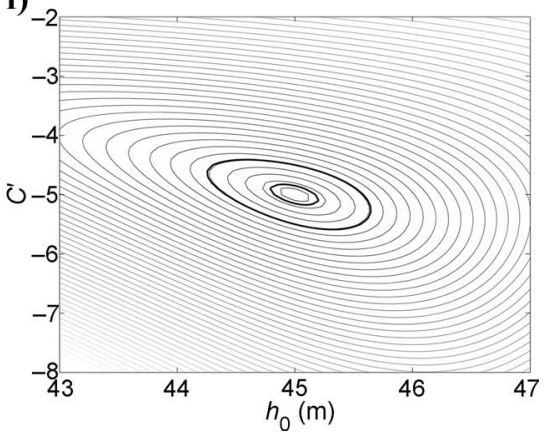

Figure 2. Error function landscape for a Gaussian water table model. (a) Drawdown amplitude/drawdown dispersion cross section near $(A, \sigma)$ $=(20,40)$. (b) Drawdown amplitude/electrokinetic coupling coefficient cross section near $\left(A, C^{\prime}\right)=(20,-5)$. (c) Drawdown dispersion/ electrokinetic coupling coefficient cross section near $\left(\sigma, C^{\prime}\right)=(40,-5)$. (d) Drawdown amplitude/reference hydraulic-head cross section near $\left(A, h_{0}\right)=(20,45)$. (e) Drawdown dispersion/reference hydraulic-head cross section near $\left(\sigma, h_{0}\right)=(40,45)$. (f) Reference hydraulichead/electrokinetic coupling coefficient cross section near $\left(h_{0}, C^{\prime}\right)=(45,-5)$. 
bounds. This depends on the value of $C^{\prime}$ found on the search, as shown on the study of the equivalences.

Figure $3 \mathrm{c}$ and $\mathrm{d}$ shows the histogram of the coupling coefficient $C^{\prime}$ and the error curve for PSO as a function of the iterations. For this synthetic example, the mode is around $-4.08 \mathrm{mV} / \mathrm{m}$ instead of the true value, $C^{\prime}=-5.00 \mathrm{mV} / \mathrm{m}$. The value is consistent with the fact that the amplitude of the best model has been underestimated. PSO reaches the region of low-misfit errors before 20 iterations (Figure $3 d)$. These inversions have been carried using $\left(\omega, a_{g}, a_{\ell}\right)=(0.8$, $2.0,1.8)$, which gives slightly more weight to the global best than to the local best. When using a cloud PSO algorithm, the convergence rate is slower (the low-misfit region is reached at 50-100 iterations) because of its bigger exploratory capabilities, but the posterior analysis provides similar results. Finally, it is possible to observe that in some inversions the global-best solution does not perfectly reproduce the true model, even in the noise-free synthetic case. This can result because (1) the parameter space has not been thoroughly searched and more algorithm iterations are needed or (2) trade-offs exist between the model parameters. In this case, the inverted $C^{\prime}$ value found is higher than the true one, causing a higher amplitude of the hydraulic head (see Figure 2b).

The algorithm also has been tested with measurements affected by noise. Figure 4 shows the results with $20 \%$ white Gaussian noise. The algorithm is quite robust in the presence of Gaussian noise with an estimate of $C^{\prime}=-3.95 \mathrm{mV} / \mathrm{m}$. The insensitivity to the Gaussian noise might be from the smoothing effect introduced in the PSO algorithm through the global best. All other features commented about for the noise-free case remain valid.

SP measurements always show the presence of outliers, mainly from measurement errors and/or heterogeneities in the ground not accounted for in the geophysical forward model. Before any inversion, it is common to filter the measurements first. With the PSO algorithm, this procedure could be avoided when Gaussian noise is present. Nevertheless, the presence of systematic and non-Gaussian noise will presumably affect the algorithm's performance; the minimum value of the misfit that can be reached will be higher because these errors cannot be taken into account by the mathematical model.

Finally, we illustrate the use of the algorithm with field data taken from work by Bogoslovsky and Ogilvy (1973), corresponding to SP data collected near a pumping well. Although the details of this experiment are not available, it is a useful data set to model because it has been studied by other workers (Revil et al., 2003; Darnet et al., 2004; Minsley et al., 2007).

Figure 5 shows the SP profile collected over the pumping well and the water-table elevations measured in several monitoring wells (inverse triangles). The broad, positive SP anomaly is fairly symmetric around the well position and, as expected with field data, some noise
Figure 3. Synthetic SP case. (a) Observed and predicted SP anomalies; no noise. (b) Synthetic water table and predicted models. (c) Histogram of inverted $C^{\prime}$ values. (d) Error curve.

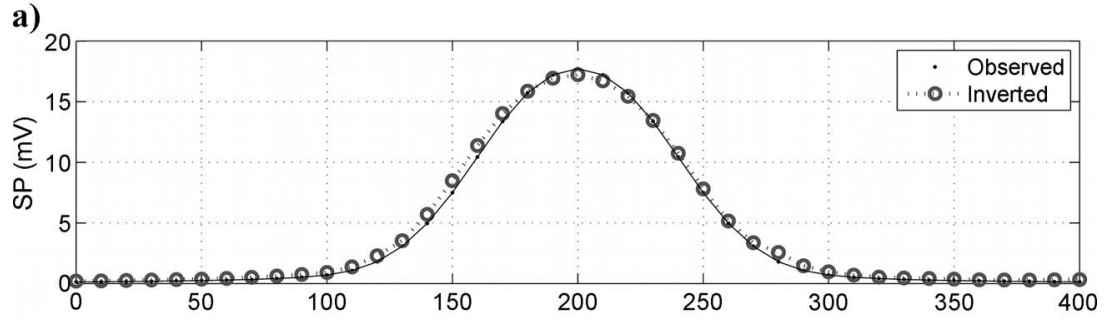

b)

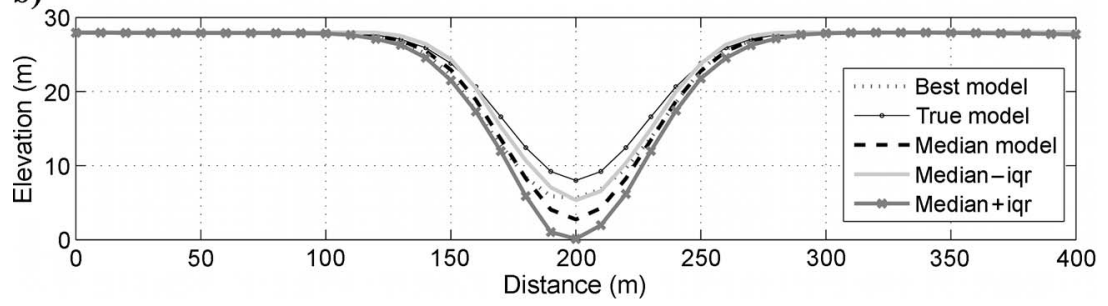

c)

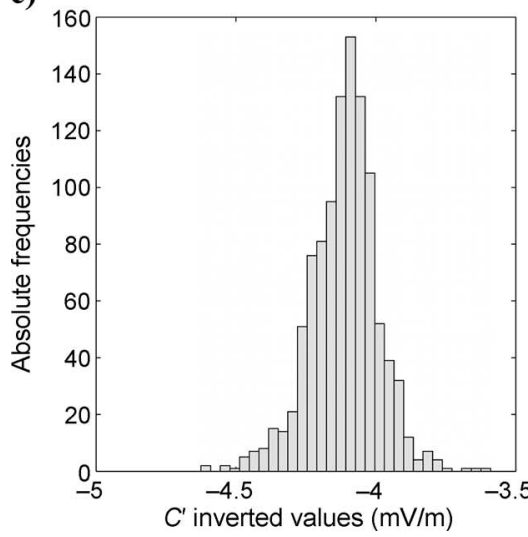

d)

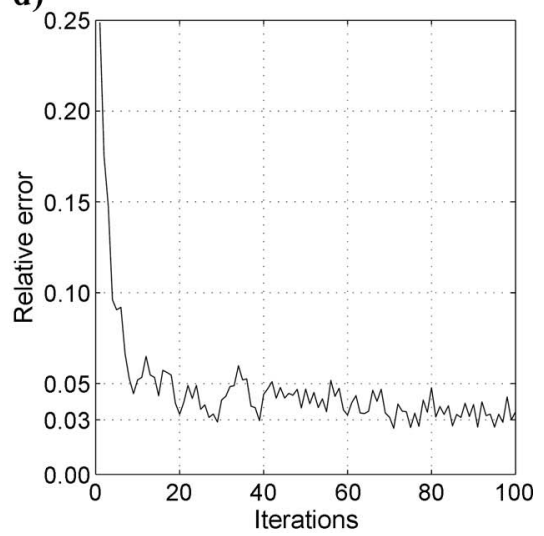


corrupts the signal. There is good agreement between the prediction of the PSO algorithm and the water table measured in the monitoring wells except at two points, which are very close to the lower and upper bounds deduced from the posterior analysis of the region on models of $20 \%$ error. The algorithm can find the region of low misfits in 30 iterations. The coupling coefficient is estimated as $C^{\prime}=$ $-13.9 \mathrm{mV} / \mathrm{m}$ (Figure $5 \mathrm{~b}$ ). This value is very close to the one obtained by Revil et al. (2003) with the simplex algorithm $\left(C^{\prime}=\right.$ $-14.2 \mathrm{mV} / \mathrm{m}$ ). Darnet et al. (2003) obtain $C^{\prime}=-0.8 \mathrm{mV} / \mathrm{m}$, applying a genetic algorithm and taking into account the high electrical conductivity of the pumping well's metal casing. Minsley et al. (2007) do not include any effects from the well casings but use a resistivity model that approximates the unsaturated drawdown to invert the SP data in terms of source locations. They did not estimate the coupling coefficient.

To obtain the model presented in Figure 5, we use a correlation length of 10 points, as suggested by the autocorrelation function of the SP data (Figure 6a). The autocorrelation function provides the spatial correlation of the SP signal considered as a 1D stochastic process. We hypothesize that the hydraulic head and the SP signal have a similar correlation length. This provides an initial guess for the hydraulic-head correlation distance that must be checked through the inversion of the SP data. In fact, the SP integral equation 4 indicates a smoothing effect of the SP anomaly with respect to the hydraulic head.

The search space and the smoothing parameter are very important in the search algorithm because the inverse solution is not unique. If the search space is poorly designed, the algorithm will not find a lowmisfit region according to the numerical priors. The search space in this case was $C^{\prime} \in[-20,-0.5]$, and the search space for the hydraulic head was designed using 15 . The $h_{0}$ parameter was fixed to $19.5 \mathrm{~m}$, based on prior knowledge. If this value is not well known, $h_{0}$ can be considered an additional parameter on the search. Figure $6 \mathrm{~b}$ shows the histogram of $h_{0}$ when the search space is $h_{0} \in[15,25]$. The histogram mode is around $20 \mathrm{~m}$.

As mentioned, the SPO inverse problem is very ill posed, i.e., it is possible to find different solutions because of the existing trade-offs between the different parameters. For instance, Figure 7 shows another solution found with 10 points of correlation length where $C^{\prime}$ is estimated as $-8.74 \mathrm{mV} / \mathrm{m}$. The final misfit for this model is a bit higher than in the previous case (around 16\%). The fact that the water table model has a higher value of $C^{\prime}$ seems to coincide with the rougher appearance found for the hydraulic head when compared to the solution in Figure 5. The correlation length is of paramount importance because it gives the water table the desired spatial correlation. It is also possible to find rougher solutions (with four or six points of correlation length) that adequately fit the SP anomaly, but a)

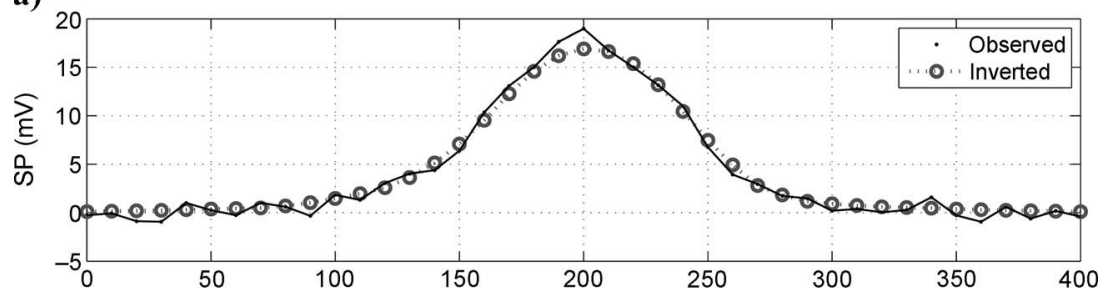

b)

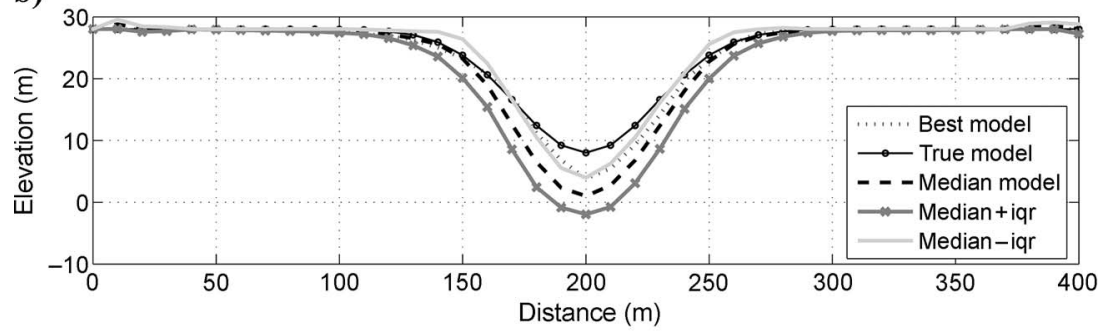

c)

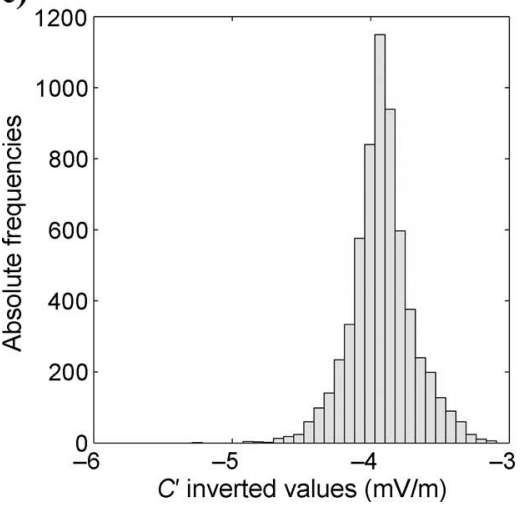

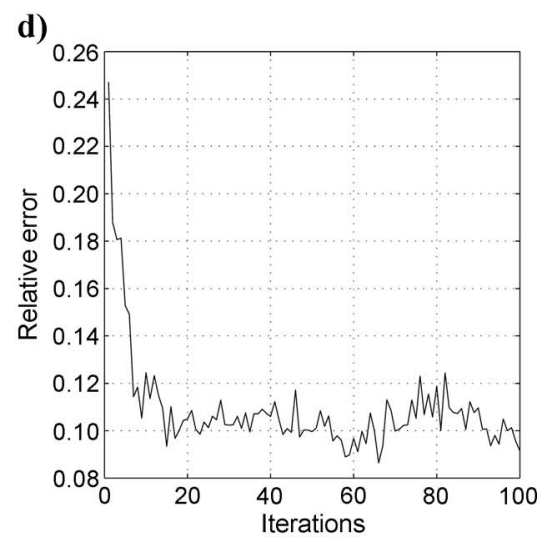

d)
Figure 4. Synthetic SP case with a 20\% Gaussian noise. (a) Observed and predicted SP anomalies. (b) Synthetic water table and predicted models. (c) Histogram of inverted $C^{\prime}$ values. (d) Error curve. 
Figure 5. Estimate of the piezometric level from SP data observed by Bogoslovsky and Ogilvy (1973) with inverted $C^{\prime}=-13.9 \mathrm{mV} / \mathrm{m}$. (a) SP observed and predicted profiles. (b) Predicted piezometric levels with lower and upper bounds. (c) Histogram of inverted $C^{\prime}$ values. (d) Error curve.

the obtained water table does not fit the borehole data very well. When the correlation length decreases below six points, most of the hydraulic-head models found by the PSO algorithm are close to the boundaries of the search space, producing results that are not geologically plausible. For all of these simulations, the coupling parameter also had its mode in the interval $[-9,-8] \mathrm{mV} / \mathrm{m}$. Taking into account these experimental results, the correlation length and the coupling coefficient influence the smooth character of the water table: roughest solutions seem to be associated with higher coupling constants. Also, both parameters are quite well resolved because no correlation length nor coupling constant provides acceptable geologic solutions. Although there is no clear physical explanation for this fact, it must be considered as another numerical illustration of the ill-posed character of the SP inverse problem.

Finally, Figure 8 compares PSO with the results obtained by other workers using the simplex method with 1000 iterations (Revil et al., 2003) and the genetic algorithm (Darnet et al., 2003). PSO and simplex give very similar results, as expected, because of the values found for the coupling coefficients. Without knowing the details of the experiment related to these SP data, such as pumping rate and resistivity distribution in the subsoil, it is difficult to calibrate and compare the results. Nevertheless, PSO seems to be very efficient in rapidly finding an acceptable solution for the water table and coupling coefficient and in exploring the space of possible solutions.

\section{FINAL DISCUSSION}

As with many other geophysical problems, the SP inverse problem is ill posed. In the absence of prior information, local methods provide unstable solutions, which depend greatly on the initial guess. In the case of streaming potential and by means of synthetic modeling, we have shown that the topography of the prediction error (cost function) for the SP inverse problem corresponds to elongated valleys with localized sinkholes when noise is present. This flat topography also indicates the existing trade-offs between the model parameters in the SP inverse problem. Thus, solving the SP inverse problem suffers from the same weakness as other inverse problems. Instabilities can be solved through local optimization and regularization techniques using good prior information. Bayesian approaches serve in these cases not only to look for the maximum a posteriori model but for the posterior distribution itself. Prior information is valuable and costly, and in many situations it is unavailable because the geophysical method is performed in a very premature phase of the hydrogeologic project and/or because the exploration is run in countries where only very-low-cost methods can be used as a result of technological and economic constraints. In these cases, sampling procedures can approach the solution and appraisal of the inverse problem. Monte Carlo methods are very good at accomplishing this task, but they also tend to be very inefficient and highly time consuming. 

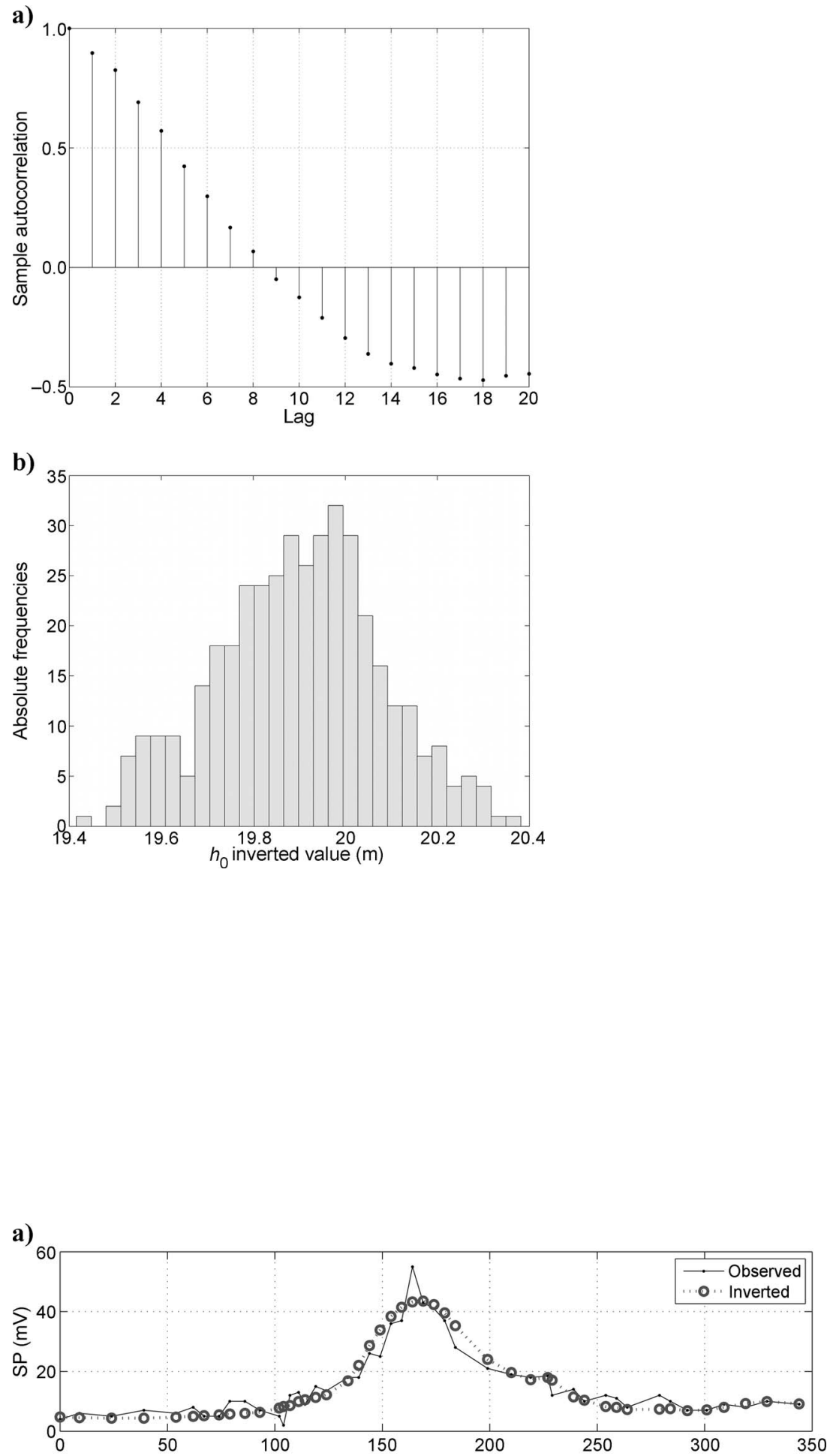

b)

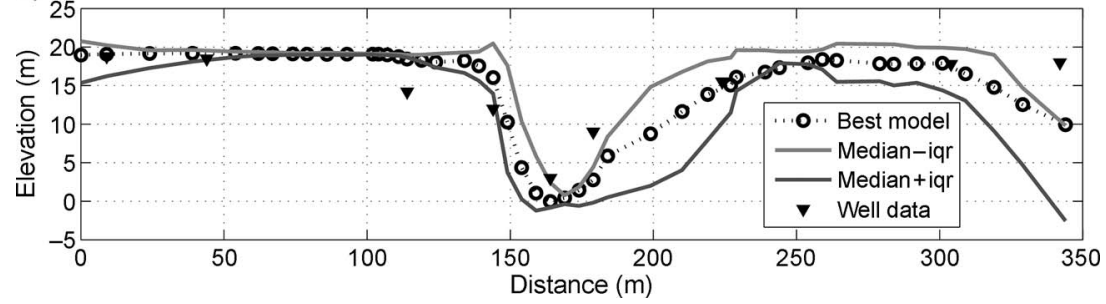

Figure 6. (a) Experimental autocorrelation function of SP data observed by Bogoslovsky and Ogilvy (1973). (b) Histogram of $h_{0}$ inverted values.

Figure 7. Another solution for the Bogoslovsky and Ogilvy (1973) SP data set. (a) SP observed and predicted profiles. (b) Predicted piezometric levels with lower and upper bounds. In this case, $C^{\prime}$ is estimated as $-8.9 \mathrm{mV} / \mathrm{m}$. 

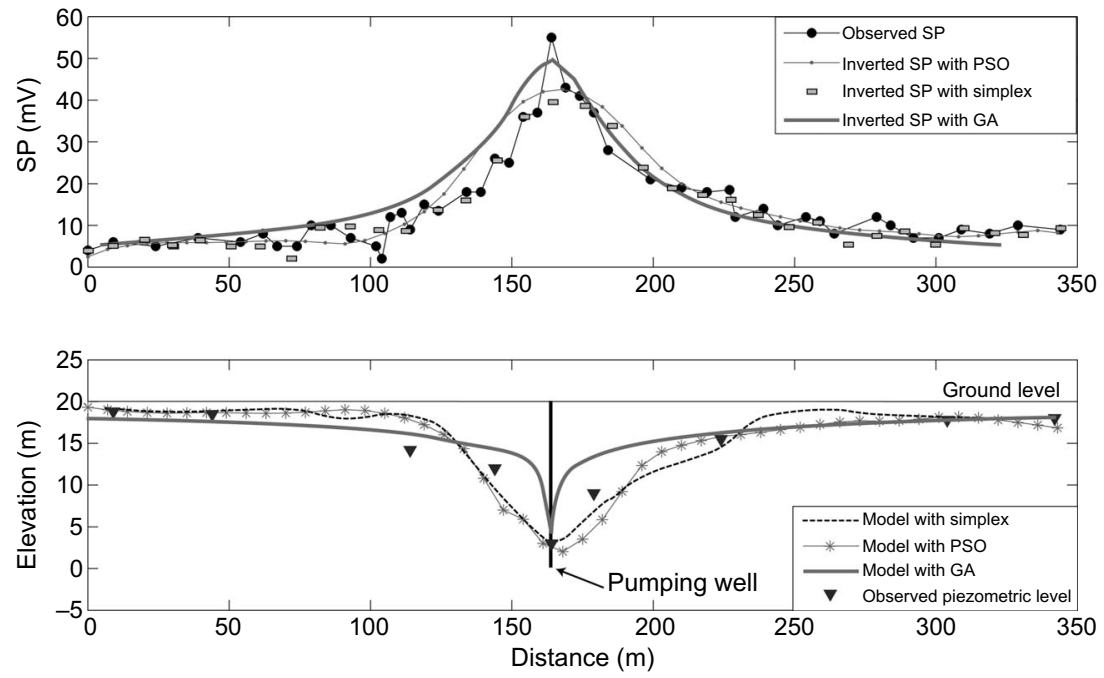

Figure 8. Comparison of PSO (Fernández-Martínez et al., 2009), simplex from Revil et al. (2003), and genetic algorithms (GA) from Darnet et al. (2003) for the Bogoslovsky and Ogilvy (1973) data set. (a) Observed and predicted data using different algorithms. (b) Inverted models and observed piezometric levels.

PSO is a simple algorithm that can find plausible solutions and sample the model space according to some minimum prior requirements: the search space and the regularity required for the hydraulichead model. PSO differentiates from other global methods in the fact that, although it is a stochastic search method, it is not heuristic. Taking into account its simplicity, robustness, and versatility (it can be used for exploitation and/or exploration purposes), we recommend that the hydrogeophysical community dealing with this kind of inverse problems consider using it. We expect that the use of global algorithms in inversion, such as PSO, will increase the reliability of the SP method in hydrogeologic investigations.

\section{ACKNOWLEDGMENTS}

This work benefited from a one-month grant given by the University of Bordeaux 1 in November 2007 and from a one-year sabbatical grant (2008-2009) at the University of California Berkeley (Department of Civil and Environmental Engineering) given by the University of Oviedo (Spain) and by the Secretaría de Estado de Universidades y de Investigación of the Spanish Ministry of Science and Innovation. We also acknowledge financial support for 2009-2010 from the University of California Berkeley, the Lawrence Berkeley National Laboratory (Earth Science Division), and the Energy Resources Engineering Department of Stanford University (Stanford Center for Reservoir Forecasting and Smart Field Consortiums). The French National Scientific Research Center and the National Institute of Universe Sciences (CNRS/NSU) are also thanked for their support. Finally, we thank Douglas Sassen (Lawrence Berkeley Laboratories, Earth Science Division) and Laura Nikravesh (UC Berkeley) for suggestions and style corrections made to this manuscript.

\section{REFERENCES}

Aster, R. C., C. H. Thurber, and B. Borchers, 2005, Parameter estimation and inverse problems: Elsevier Academic Press.

Bigalke, J., and E. W. Grabner, 1997, The geobattery model: A contribution to large-scale electrochemistry: Electrochimica Acta, 42, 3443-3452.
Birch, F. S., 1998, Imaging the water table by filtering self-potential profiles: Ground Water, 36, 779-782.

Bogoslovsky, V. V., and A. A. Ogilvy, 1973, Deformations of natural electric fields near drainage structures: Geophysical Prospecting, 21, 716-723.

Bolève, A., A. Revil, F. Janod, J. L. Mattiuzzo, and A. Jardani, 2007, Forward modeling and validation of a new formulation to compute self-potential signals associated with ground water flow: Hydrology and Earth System Sciences, 11, 1661-1671.

Carlisle, A., and G. Dozier, 2001, An off-the-shelf PSO: Proceedings of the Workshop on Particle Swarm Optimization, 1-6.

Clerc, M., and J. Kennedy, 2002, The particle swarmexplosion, stability, and convergence in a multidimensional complex space: IEEE Transactions on Evolutionary Computation, 6, 58-73.

Darnet, M., A. Maineult, and G. Marquis, 2004, On the origins of self potential (SP) anomalies induced by water injections into geothermal reservoirs: Geophysical Research Letters, 31, L19609.

Darnet, M., G. Marquis, and P. Sailhac, 2003, Estimating aquifer hydraulic properties from the inversion of surface streaming potential (SP) anomalies: Geophysical Research Letters, 30, 1679

Davis, J. A., R. O. James, and J. Leckie, 1978, Surface ionization and complexation at the oxide/water interface - I. Computation of electrical double layer properties in simple electrolytes: Journal of Colloid and Interface Science, 63, 480-499.

Fernández Álvarez, J. P., J. L. Fernández Martínez, and C. O. Menéndez Pérez, 2008, Feasibility analysis of the use of binary genetic algorithms as importance samplers application to a geoelectrical VES inverse problem: Mathematical Geosciences, 40, 375-408.

Fernández-Martínez, J. L., J. P. Fernández-Álvarez, E. García-Gonzalo, C. O. Menéndez-Pérez, and H. A. Kuzma, 2008a, Particle swarm optimization (PSO): A simple and powerful algorithm family for geophysical inversion: 78th Annual International Meeting, SEG, Expanded Abstracts, 3568-3571.

Fernández Martínez, J. L., and E. García-Gonzalo, 2008, The generalized PSO: A new door to PSO evolution: Journal of Artificial Evolution and Applications, 2008, 861275

, 2009, The PSO family: Deduction, stochastic analysis and comparison: Swarm Intelligence, 3, 245-273.

Fernández-Martínez, J. L., E. García-Gonzalo, and J. P. Fernández-Alvarez, 2008b, Theoretical analysis of particle swarm trajectories through a mechanical analogy: International Journal of Computational Intelligence Research, 4, 93-104.

Fernández-Martínez, J. L., H. Kuzma, E. García-Gonzalo, J. M. FernándezDíaz, J. P. Fernández-Álvarez, and C. O. Menéndez-Pérez, 2009, Application of global optimization algorithms to a salt water intrusion problem: Symposium on the Application of Geophysics to Engineering and Environmental Problems (SAGEEP), 22, 252-260.

Fernández-Martínez, J. L., and L. M. Pedruelo-González, 2008, Anisotropic mean traveltime curves: A method to estimate anisotropic parameters from 2D transmission tomographic data: Mathematical Geosciences, 41, no. 2 , $163-192$.

Fitterman, D. V., 1978, Electrokinetic and magnetic anomalies associated with dilatant regions in a layered earth: Journal of Geophysical Research, 83, 5923-5928.

Fournier, C., 1989, Spontaneous potentials and resistivity surveys applied to hydrogeology in a volcanic area: Case history of the Chaine des Puys (Puy-de-Dôme, France): Geophysical Prospecting, 37, 647-668.

García-Gonzalo, E., and J. L. Fernández Martínez, 2009, Design of a simple and powerful particle swarm optimizer: Proceedings of the International Conference on Computational and Mathematical Methods in Science and Engineering, 4, 1280-1290.

Gibert, D., and M. Pessel, 2001, Identification of sources of potential fields with the continuous wavelet transform: Application to SP profiles: Geophysical Research Letters, 28, 1863-1866.

Guichet, X., L. Jouniaux, and N. Catel, 2006, Modification of streaming potential by precipitation of calcite in a sand-water system: Laboratory measurements in the $\mathrm{pH}$ range from 4 to 12: Geophysical Journal International, 166, 445-460.

Holland, J. H., 1992, Adaptation in natural and artificial systems: Massachusetts Institute of Technology Press.

Ishido, T., and H. Mizutani, 1981, Experimental and theoretical basis of electrokinetic phenomena in rock-water systems and its application to geophysics: Journal of Geophysical Research, 86, 1763-1775.

Jardani, A., A. Revil, W. Barrash, A. Crespy, E. Rizzo, S. Straface, M. Cardiff, B. Malama, C. Miller, and T. Johnson, 2009, Reconstruction of the 
water table from self-potential data: A Bayesian approach: Ground Water, 47, 213-227.

Kennedy, J., and R. C. Eberhart, 1995, Particle swarm optimization: Proceedings of the IEEE International Conference on Neural Networks, 4 1942-1948.

Kirkpatrick, S., C. D. Gelatt, and M. P. Vecchi, 1983, Optimization by simulated annealing: Science, 220, 671-680.

Linde, N., A. Revil, A. Bolève, C. Dagès, J. Castermant, B. Suski, and M. Voltz, 2007, Estimation of the water table throughout a catchment using self-potential and piezometric data in a Bayesian framework: Journal of Hydrology, 334, 88-98.

Ma, X. Q., 2002, Simultaneous inversion of prestack seismic data for rock properties using simulated annealing: Geophysics, 67, 1877-1885.

Maineult, A., Y. Bernabé, and P. Ackerer, 2004, Electrical response of flow, diffusion and advection in a laboratory sand-box: Vadose Zone Journal, 3, $1180-1192$

Maineult, A., E. Strobach, and J. Renner, 2008, Self-potential signals induced by periodic pumping tests: Journal of Geophysical Research, 113 B01203

Minsley, B., 2007, Modeling and inversion of self-potential data: Ph.D. dissertation, Massachusetts Institute of Technology.

Minsley, B. J., J. Sogade, and F. D. Morgan, 2007, Three-dimensional source inversion of self-potential data: Journal of Geophysical Research, 112 B02202.

Morgan, F. D., E. R. Williams, and T. R. Madden, 1989, Streaming potential properties of Westerly Granite with applications: Journal of Geophysical Research, 94, 12449-12461

Mosegaard, K., and A. Tarantola, 1995, Monte Carlo sampling of solutions to inverse problems: Journal of Geophysical Research, 100, 12431-12447.

Naudet, V., J. L. Fernández-Martínez, E. García-Gonzalo, and J. P. Fernández-Álvarez, 2008, Estimation of water table from self-potential data using particle swarm optimization (PSO): 78th Annual International Meeting, SEG, Expanded Abstracts, 1203-1207.

Naudet, V., A. Revil, E. Rizzo, J. Y. Bottero, and P. Bégassat, 2004, Groundwater redox conditions and conductivity in a contaminant plume from geoelectrical investigations: Hydrology and Earth System Sciences, 8, 8-22.

Onsager, L., 1931, Reciprocal relations in irreversible processes, I: Physical Review, 37, 405-426.

Parker, R. L., 1994, Geophysical inverse theory: Princeton University Press.

Patella, D., 1997, Introduction to ground surface self-potential tomography: Geophysical Prospecting, 45, 653-681.

Poli, R., 2008, Analysis of the publications on the applications of particle swarm optimization: Journal of Artificial Evolution and Applications, 2008, 685175

Revil, A., V. Naudet, and J. D. Meunier, 2004, The hydroelectric problem of porous rocks: Inversion of the position of the water table from self-potential data: Geophysical Journal International, 159, 435-444.

Revil, A., V. Naudet, J. Nouzaret, and M. Pessel, 2003, Principles of electrography applied to self-potential electrokinetic sources and hydrogeological applications: Water Resources Research, 39, 1114-1129.

Rizzo, E., B. Suski, A. Revil, S. Straface, and S. Troisi, 2004, Self-potential signals associated with pumping tests experiments: Journal of Geophysical Research, 109, B10203.

Sailhac, P., and G. Marquis, 2001, Analytic potentials for the forward and inverse modeling of SP anomalies caused by subsurface fluid flow: Geophysical Research Letters, 28, 1851-1854.

Sambridge, M., 1999a, Geophysical Inversion with a neighbourhood algorithm - I. Searching a parameter space: Geophysical Journal International, 138, 479-494.

-, 1999b, Geophysical inversion with a neighbourhood algorithm - II. Appraising the ensemble: Geophysical Journal International, 138, 727-746.

Sanyi, Y., W. Shangxu, and T. Nan, 2009, Swarm intelligence optimization and its application in geophysical data inversion: Applied Geophysics, 6 , 166-174.

Scales, J. A., and L. Tenorio, 2001, Prior information and uncertainty in inverse problems: Geophysics, 66, 389-397.

Sen, M. K., and P. L. Stoffa, 1995, Global optimization methods in geophysical inversion: Elsevier Scientific Publ. Co., Inc.

Shaw, R., and S. Srivastava, 2007, Particle swarm optimization: A new tool to invert geophysical data: Geophysics, 72, no. 2, F75-F83.

Sheffer, M. R., and D. W. Oldenburg, 2007, Three-dimensional modelling of streaming potential: Geophysical Journal International, 169, 839-848.

Stoffa, P. L., and M. K. Sen, 1991, Nonlinear multiparameter optimization using genetic algorithm: Inversion of plane-wave seismograms: Geophysics, 56, 1794-1810.

Storn, R., and K. Price, 1997, Differential evolution - A simple and efficient heuristic for global optimization over continuous spaces: Journal of Global Optimization, 11, 341-359.

Tarantola, A., 2004, Inverse problem theory and model parameter estimation: Society for Industrial and Applied Mathematics.

Trelea, I. C., 2003, The particle swarm optimization algorithm: Convergence analysis and parameter selection: Information Processing Letters, 85 , $317-325$. 